\title{
Effects of dopamine on retinal and choroidal blood flow parameters in humans
}

\author{
Karl-Heinz Huemer, Claudia Zawinka, Gerhard Garhöfer, Elisabeth Golestani, Brigitte Litschauer, \\ Guido T Dorner, Leopold Schmetterer
}

Br J Ophthalmol 2007;91:1194-1198. doi: 10.1136/bjo.2006.113399

See end of article for authors' affiliations

......................

Correspondence to: Leopold Schmetterer, Department of Clinical

Pharmacology, Währinger Gürtel 18-20, A-1090

Vienna/Austria; leopold. schmetterer@meduniwien. ac.at

Accepted 1 March 2007

Published Online First

23 March 2007

\begin{abstract}
Aim: To investigate the effect of dopamine on retinal and choroidal blood flow in humans.
Methods: We investigated the effect of two doses of intravenous dopamine ( 5 and $10 \mu \mathrm{g} / \mathrm{kg} / \mathrm{min}$ ) via a randomised double-masked crossover study in 12 healthy subjects chosen from a total of 16 . Blood flow parameters in retina, optic nerve head and choroid were assessed with bi-directional laser Doppler velocimetry, laser Doppler flowmetry and laser interferometric measurement of fundus pulsation amplitude, respectively.

Results: Intravenous dopamine dose-dependently increased retinal blood cell velocity and fundus pulsation amplitude $(p<0.001)$. At the highest administered dose red blood cell velocity in retinal vessels increased by $37 \%$ and fundus pulsation amplitude by $24 \%$. By contrast, optic nerve head blood flow did not change with dopamine administration.

Conclusions: Our data indicate that dopamine has a pronounced enhancing effect on the retinal perfusion in humans. Further studies are required to establish the exact role of dopamine in the regulation of choroidal and optic nerve head blood flow.
\end{abstract}

1 $\mathrm{t}$ has been demonstrated that dopamine antagonists increase ocular blood flow in rabbits. ${ }^{1}$ Other dopamine antagonists had similar effects whereas no effect of dopamine agonists was found on pulsatile ocular blood flow. ${ }^{2}$ In addition, dopamine has been shown to play a role in intraocular pressure (IOP) control. ${ }^{3}$ We have shown in a previous study that dopamine increases retinal vessel diameter and reduces flickerinduced retinal vessel changes. ${ }^{5}$ Based on the results of this preceding study, we hypothesised that dopamine might increase retinal blood flow in healthy humans. In the present study we measured blood flow velocity in retinal vessels during dopamine infusion to allow for calculation of dopamineinduced effects on retinal blood flow. In addition, we examined the effect of dopamine on blood flow parameters in the optic disc and choroid.

\section{METHODS \\ Subjects}

The study was performed in 16 healthy male subjects aged between 22 and 34 years. All subjects had to pass a medical examination including physical status, ECG, blood count, blood chemistry, urine screening test and drug screening. In addition, an ophthalmological examination was performed. Subjects were excluded from the study if any possibly interfering abnormalities were present. To be included in this study, ametropias had to be less than 3 diopters and no anisometropia of more than 1 diopter was allowed. Subjects had to refrain from alcohol, caffeine and other stimulating methylxanthine containing nutrients for $12 \mathrm{~h}$ prior to each study day.

The study protocol was approved by the Ethics Committee of the University of Vienna School of Medicine and was performed in adherence with the Declaration of Helsinki. All subjects signed a written informed consent before being included in this study.

\section{Study design}

The study was performed following a randomised, doubleblinded, placebo and time-controlled, two way crossover design. Intravenous canullae were positioned on both forearms for administering saline or dopamine hydrochloride on one side and for withdrawing blood for dopamine measurement on the other arm.

Placebo or dopamine hydrochoride was administered on two separate study days using identical protocols. All measurements were performed in a sitting position. Subjects remained seated in a moveable chair and were positioned in front of the different devices. On each day we performed four measurement cycles. The first cycle was used to adjust instruments and to select appropriate measurement locations. The data from this first step were not used for analysis. During the following measurement cycles a continuous intravenous infusion was administered, starting with physiological saline solution representing the baseline value. In two consecutive cycles 5 or $10 \mu \mathrm{g} /$ $\mathrm{kg} / \mathrm{min}$ of dopamine hydrochloride was administered. Each measurement cycle consisted of withdrawing a blood sample for determining plasma dopamine levels, measurements of the IOP, blood flow velocity in the ophthalmic artery (OA) with Doppler sonography, retinal blood velocity with bi-directional laser Doppler velocimetry (LDV), optic nerve head (ONH) blood flow with the laser Doppler Flowmetry and fundus pulsation amplitude (FPA) with laser interferometric fundus pulsation measurements. Measurement was started $12 \mathrm{~min}$ after the beginning of each infusion step keeping the same sequence in all cycles. After all measurements of one cycle (about $30 \mathrm{~min}$ after onset of each dose) were completed the next infusion step was started. After finishing the last measurement cycle infusion was discontinued. The washout period between study days was at least 7 days.

\section{Medications, administration of dopamine}

The pupil of the eye used for measurements was dilated with tropicamide eye drops (Mydriaticum Agepha ${ }^{\circledR}$, Agepha, Vienna,

Abbreviations: FPA, fundus pulsation amplitude; IOP, intraocular pressure; LDF, laser Doppler flowmetry; LDV, (bi-directional) laser Doppler velocimetry; $\mathrm{OA}$, ophthalmic artery; $\mathrm{ONH}$, optic nerve head; $\mathrm{PR}$, pulse rate; $\mathrm{SBP}$, systolic blood pressure 
Table 1 Baseline values (mean \pm SD)

\begin{tabular}{lcc}
\hline & Placebo day & Dopamine day \\
\hline Systolic blood pressure $(\mathrm{mmHg})$ & $118.3 \pm 9.1$ & $116.6 \pm 8.6$ \\
Diastolic blood pressure $(\mathrm{mmHg})$ & $62.1 \pm 5.9$ & $61.5 \pm 5.3$ \\
Mean arterial pressure $(\mathrm{mmHg})$ & $81.4 \pm 6.4$ & $80.1 \pm 5.4$ \\
Pulse rate (min) & $66.3 \pm 10.3$ & $68.2 \pm 11.3$ \\
Intraocular pressure (mmHg) & $13.2 \pm 2.0$ & $13.4 \pm 2.7$ \\
Dopamine plasma levels $(\mathrm{ng} / \mathrm{l})$ & $43 \pm 31$ & $45 \pm 33$ \\
PSV (cm/s) & $43.2 \pm 5.3$ & $42.5 \pm 5.5$ \\
EDV (cm/s) & $8.1 \pm 1.6$ & $7.8 \pm 1.4$ \\
MFV (cm/s) & $17.3 \pm 3.3$ & $18.0 \pm 3.6$ \\
Optic nerve head blood flow (units) & $6.9 \pm 1.6$ & $6.7 \pm 1.6$ \\
Fundus pulsation amplitude $(\mu \mathrm{m})$ & $3.9 \pm 1.1$ & $3.8 \pm 1.2$ \\
RBV (cm/s) & $2.1 \pm 0.6$ & $2.0 \pm 0.5$ \\
\hline
\end{tabular}

EDV, end diastolic velocity; MFV, mean flow velocity; PSV, peak systolic velocity; RBV, blood velocity in retinal veins.

Austria). Dopamine hydrochloride (Dopamin Giulini ${ }^{\circledR}$, Solvay Pharmaceuticals, Hannover, Germany) was administrated intravenously with a peristaltic pump. The doses were 5 or $10 \mu \mathrm{g} / \mathrm{kg} / \mathrm{min}$ at a concentration of $0.4 \mathrm{mg} / \mathrm{ml}$ in saline solution. For placebo control physiological saline solution was used.

\section{Measurements}

Dopamine plasma levels were determined by high performance liquid chromatography with electrochemical detection..$^{5-7}$ Interassay coefficients of variation were less than 5\%. Brachial artery systolic, diastolic and mean blood pressure was monitored automatically every $5 \mathrm{~min}$ and pulse rate (PR) was monitored continuously with a finger pulse oximeter using a Hewlett Packard Model 66S Monitor (Palo Alto, USA). IOP was measured with standard applanation tonometry after local application of fluorescein and oxybuprocain eyedrops.

Cardiac synchronous fundus pulsations were assessed by laser interferometry by focussing the laser spot to the fovea. The FPA is the maximum distance change between cornea and retina during the cardiac cycle and estimates the pulsatile choroidal blood flow. ${ }^{8}{ }^{9}$

Peak systolic flow velocity and minimal diastolic flow velocity of the OA were assessed with a $3.25 \mathrm{MHz}$ probe with pulsed Doppler device $^{10}$ (CFM 750, Vingmed Sound, Horten, Norway). The OA was measured anteriorly at the point where it crosses the optic nerve. From these parameters the mean flow velocity ( = integral of the Doppler curve/duration of the cardiac cycle) was calculated.

Using the Oculix 4000 System (Oculix Sarl, Arbaz, Switzerland) the maximum Doppler shift corresponding to the centreline erythrocyte velocity $\left(\mathrm{V}_{\max }\right)^{11}$ was measured bidirectionally. With this method the absolute velocity in the retinal vessels can be obtained. Measurements were taken from retinal veins between 1 and 2 disc diameters from the optical disc. Hence, these measurements were performed in similar regions as vessel diameters in our preceding study. ${ }^{5}$

Measurement of ONH blood flow was performed with laser Doppler flowmetry (LDF, Oculix 4000). ${ }^{12}$ In the present study, laser Doppler flowmetry was performed at the temporal neuroretinal rim of the optic disc in a location showing no visible vessels.

\section{Data analysis}

For statistical analyses we used the Statistica V.5.0 software package (Statsoft, Tulsa, Oklahoma, USA). A two-way repeated measures multivariate analysis of variance test (MANOVA) model was used for calculation of significances. Post-hoc testing was performed using planned comparisons. A twotailed $\mathrm{p}<0.05$ was considered the level of significance. Data are presented as means $\pm \mathrm{SD}$.

\section{RESULTS}

\section{Adverse effects of drug administration}

Not all subjects tolerated dopamine hydrochloride administration well at the administrated doses. At a dose of $10 \mu \mathrm{g} / \mathrm{kg} / \mathrm{min}$, six of the subjects reported nausea of varying intensity. In some cases, intense symptoms occurred only transiently after onset of the higher dose and then improved. If symptoms did not cease, we discontinued the experiment. For this reason, four subjects were not included in the statistical analysis.

\section{Dopamine plasma levels}

Dopamine plasma levels at baseline were $40 \pm 28 \mathrm{ng} / \mathrm{l}$ and $45 \pm 33 \mathrm{ng} / \mathrm{l}$ at the two study days, respectively (see table 1 , $\mathrm{p}<0.001$ versus placebo, ANOVA). On the placebo day plasma levels did not change and remained stable over the infusion period. During administration of dopamine hydrochloride the dopamine plasma levels increased dose dependently (see table 2).

\section{Effects of dopamine on systemic haemodynamics and ocular pressure}

Dopamine hydrochloride caused a significant increase in systolic blood pressure $(\mathrm{SBP})(\mathrm{p}=0.015$ versus placebo, ANOVA). At a dose of $10 \mu \mathrm{g} / \mathrm{kg} / \mathrm{min}$ SBP was significantly increased by $+15.2 \%$. Diastolic and mean arterial pressure remained constant with infusion of dopamine. With PR there was a wide inter-individual variability and some subjects showed a considerable increase during infusion of dopamine. However, the overall increase in pulse rate was not significant versus placebo.

IOP yielded values in the normal range (table 2) and did not show changes after placebo or dopamine administration.

\section{Effects of dopamine on OA}

Baseline values of velocity data are shown in table 1 (see also fig 1). Dopamine caused a significant dose-dependent increase in peak systolic flow velocity $(\mathrm{p}<0.001$ versus placebo, ANOVA), which was $38 \pm 35 \%$ at a dose of $10 \mu \mathrm{g} / \mathrm{kg} / \mathrm{min}$. By contrast, end diastolic flow velocity did not change after administration. Mean flow velocity showed an increase after infusion of dopamine ( $p=0.013$ versus placebo, ANOVA), which was, however, less pronounced than the increase in peak systolic velocity ( $18 \pm 31 \%$ at $10 \mu \mathrm{g} / \mathrm{kg} / \mathrm{min}$ ).

\section{Effects of dopamine on retinal, choroidal and $\mathrm{ONH}$ blood flow}

Whereas blood velocity in retinal veins was not changed during placebo infusion, dopamine administration increased blood velocity by $11 \pm 14 \%$ and $37 \pm 28 \%$ at 5 and $10 \mu \mathrm{g} / \mathrm{kg} / \mathrm{min}$, respectively $(\mathrm{p}<0.01$ versus placebo, ANOVA; see fig 1$)$. By contrast, blood flow in the ONH as assessed with LDF showed no significant changes between the two study days. The ocular FPA dose dependently increased with infusion of dopamine $(\mathrm{p}<0.001$ versus placebo, ANOVA). At $10 \mu \mathrm{g} / \mathrm{kg} / \mathrm{min}$ this increase was $24 \pm 23 \%$.

\section{DISCUSSION}

Animal studies of the role of dopamine in the control of ocular blood flow are inconsistent. Dopamine antagonists have been shown to increase ocular blood flow in rabbit ${ }^{13}$ whereas dopamine agonists had no effect. ${ }^{2}$ There is also evidence for dopamine increasing choroidal blood flow. ${ }^{14}{ }^{21}$ In humans, a dopamine agonist induced a decrease of cerebral blood flow. ${ }^{15}$

Consequently, comprehending the role of dopamine is entangled by differences in methodology used to assess blood flow, vascular supply divergences between the species examined as well as by the different dopamine and other 
Table 2 Systemic haemodynamic parameters, IOP and dopamine level changes during dopamine and placebo administration (mean \pm SD)

\begin{tabular}{lccc}
\hline & $\mathbf{0} \boldsymbol{\mu g} / \mathbf{k g} / \mathbf{m i n}$ & $\mathbf{5} \boldsymbol{\mu g} / \mathbf{k g} / \mathbf{m i n}$ & $10 \mu \mathbf{g} / \mathbf{k g} / \mathbf{m i n}$ \\
\hline Dopamine & & & \\
Systolic blood pressure $(\mathrm{mmHg})$ & $116.6 \pm 8.6$ & $120.4 \pm 17.5$ & $134.8 \pm 13.9$ \\
Diastolic blood pressure $(\mathrm{mmHg})$ & $61.5 \pm 5.3$ & $55.4 \pm 9.1$ & $63.3 \pm 14.8$ \\
Mean arterial pressure $(\mathrm{mmHg})$ & $80.1 \pm 5.4$ & $76.8 \pm 9.1$ & $85.6 \pm 13.2$ \\
Pulse rate (min) & $68.2 \pm 11.3$ & $75.9 \pm 11.1$ & $81.8 \pm 21.5$ \\
Intraocular pressure (mmHg) & $13.4 \pm 2.7$ & $12.5 \pm 2.5$ & $13.6 \pm 2.8$ \\
Dopamine plasma levels (ng/l) & $45 \pm 33$ & $33808 \pm 18650$ & $61650 \pm 21260$ \\
Placebo & & $118.4 \pm 9.5$ & $116.3 \pm 8.9$ \\
Systolic blood pressure (mmHg) & $118.3 \pm 9.1$ & $63.1 \pm 6.0$ & $61.4 \pm 5.8$ \\
Diastolic blood pressure (mmHg) & $62.1 \pm 5.9$ & $81.8 \pm 7.1$ & $79.4 \pm 7.2$ \\
Mean arterial pressure (mmHg) & $81.4 \pm 6.4$ & $67.3 \pm 10.1$ & $64.2 \pm 9.5$ \\
Pulse rate (min) & $66.3 \pm 10.3$ & $13.0 \pm 2.2$ & $12.6 \pm 2.3$ \\
Intraocular pressure (mmHg) & $13.2 \pm 2.5$ & $45 \pm 32$ & $49 \pm 38$ \\
Dopamine plasma levels (ng/l) & $43 \pm 31$ & & \\
\hline
\end{tabular}

catecholaminergic receptor subtypes involved. Dl receptor type mediated vasodilatory effects have been described mainly for renal and mesenterial vascular beds. ${ }^{16}$ Such dopaminergic vascular effects are often obscured by its $\beta$ adrenergic receptor-mediated inotropic actions and in higher concentrations by enhancing vasoconstriction via $\alpha$ adrenoceptors. ${ }^{17}$ In this paper, we present evidence for dopaminergic effects on different haemodynamic parameters in human ocular blood flow in vivo.

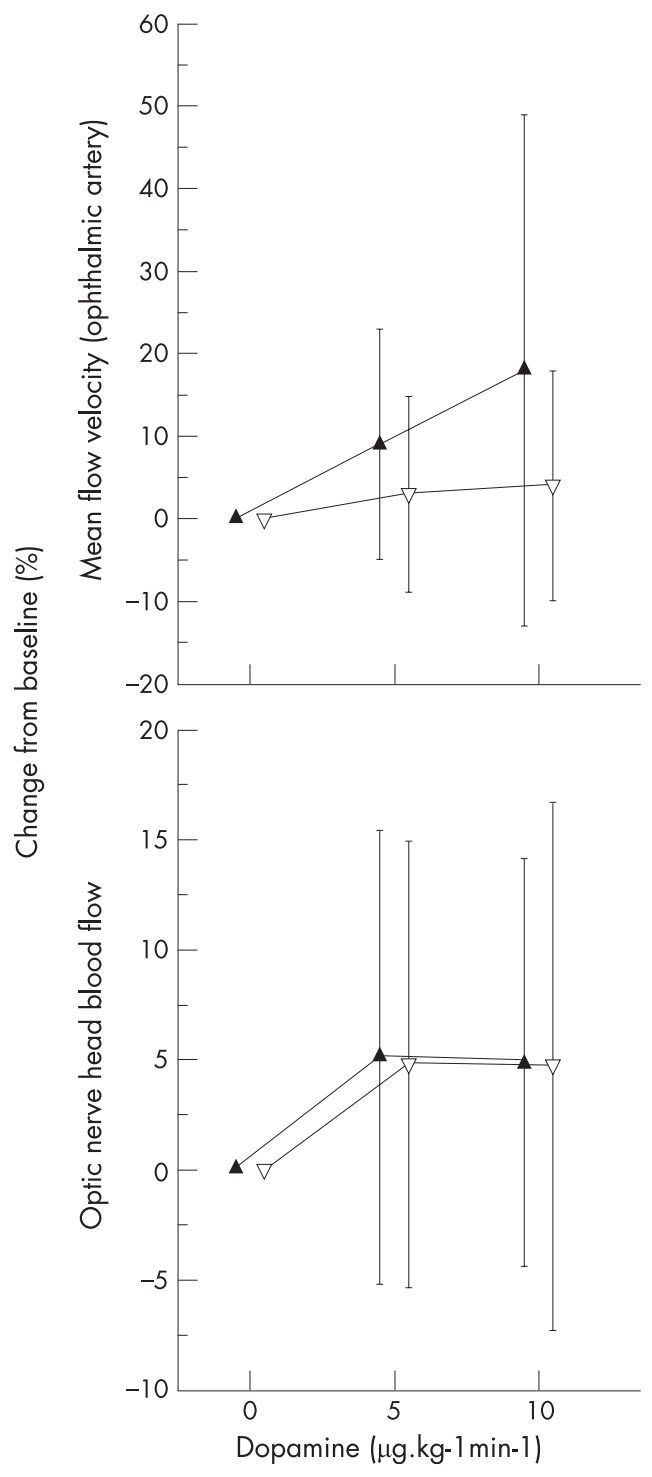

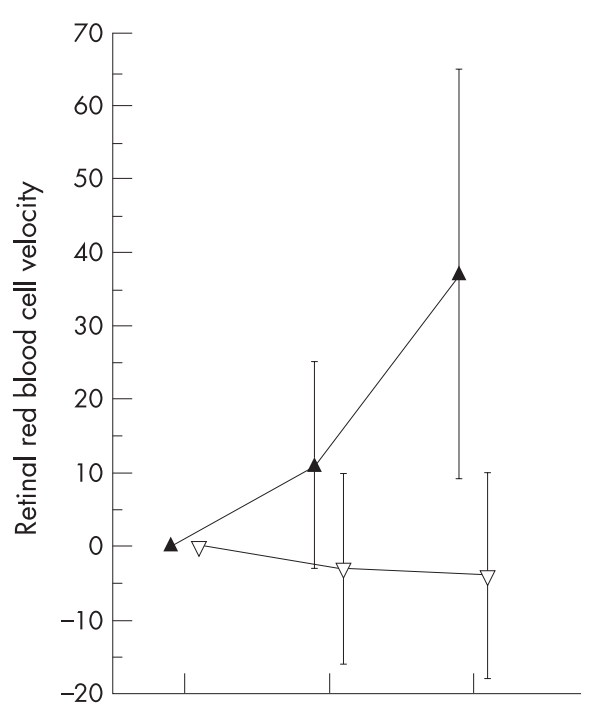

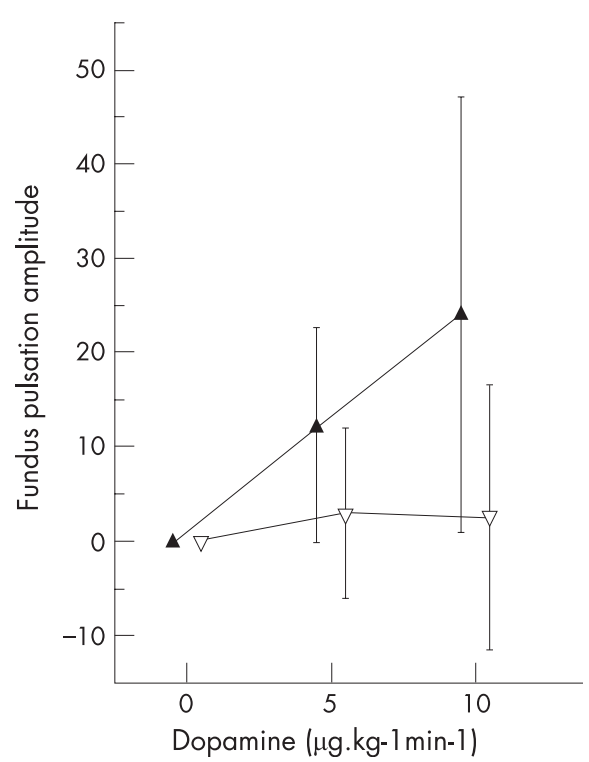

Figure 1 Effects of dopamine administration on ocular haemodynamic parameters. Solid up triangles indicate dopamine administration and open down triangles indicate placebo administration. Data are presented as means $\pm S D(n=12)$. 


\section{Dopaminergic effects on retinal blood velocity and retinal blood flow}

We found a highly significant increase in our primary outcome parameter, the retinal venous blood velocity. This increase was approximately $35 \%$ at the higher dose of $10 \mu \mathrm{g} / \mathrm{kg} / \mathrm{min}$.

We have shown earlier that dopamine increases the vessel diameter of retinal arteries and veins. ${ }^{5}$ This diameter increase was $3.8 \pm 1.1 \%$ in large branches of retinal arteries and $2.9 \pm 0.9 \%$ in retinal veins at a dose of $10 \mu \mathrm{g} / \mathrm{kg} / \mathrm{min}$. In the present study we did not repeat the vessel diameter measurements for two reasons: on the one hand data were available from our previous study that followed the same protocol in drug administration. On the other hand, and more importantly, the time schedule of measurements in the present trial was dense and additional measurement of vessel diameter would have made the study unreasonably more exhausting for the participating volunteers.

The volumetric blood flow rate in the retina is $Q=0.25 \pi \mathrm{D}^{2}$ $\left(1.6\left(V_{\max }\right)\right)$, where $\mathrm{D}$ is the vessel diameter and $\left(1.6\left(\mathrm{~V}_{\max }\right)\right)$ is the corrected mean blood velocity as calculated from the maximal centreline velocity measured with LDV. ${ }^{11}$ Applied to our data this would indicate a retinal blood flow increase of approximately $45 \%$ in response to $10 \mu \mathrm{g} / \mathrm{kg} / \mathrm{min}$ of dopamine. The tonical modulation of larger arterial and venous branch vessels by dopamine is implicated by the diameter changes we reported in our earlier study. ${ }^{5}$ The pronounced increase in blood velocity, however, indicates the involvement of additional most probably precapillary vascular regions in these dopaminedriven resistance changes. Capillary regions could also contribute to the haemodynamic effects of dopamine. Accordingly, in rats a high density of $\mathrm{Dl}$ receptor antibodies in retinal capillaries has been shown, ${ }^{18}$ and patch-clamp studies proved the functional coupling of dopamine Dl receptors to retinal vessel pericyte potassium channels. ${ }^{19}$

A general autonomic effect of dopamine on retinal blood flow is unlikely to significantly contribute to our results. Changes in systemic blood pressure were small in the present study and are unlikely to influence retinal vessel diameters. However, we have shown earlier that tyramine induced systemic blood pressure increases do not always affect retinal vessel diameters and red blood cell velocities. In addition, an indirect effect of dopamine via $\alpha$ and/or $\beta$ receptors is unlikely because even high plasma levels of noradrenaline do not alter retinal vessel diameters. ${ }^{20}$

\section{Effects on choroidal and ONH blood flow}

The effects of dopamine on fundus pulsation indicate that the pulsatile component of choroidal blood flow is also modulated by dopamine. This is in accordance with data published in rabbits, where even at relatively lower doses an increase in choroidal blood flow was observed. ${ }^{21}$ When interpreting our data of FPA measurement one needs, however, to consider that dopamine increased the pulse pressure amplitude and increased peak systolic velocity in the OA more than the end diastolic velocity. Accordingly, the increase in FPA might also represent an increase in flow pulsatility rather than in blood flow. ${ }^{22}$ Further studies using additional technology for the assessment of choroidal blood flow parameters is required to clarify this issue. Most importantly, LDF in the submacular choroid should be performed in future studies with dopamine administration. This technique was not included in the present protocol because of the tight time schedule. Comparison of such data with the FPA data obtained in the present trial could help to elucidate the role of dopamine in choroidal blood flow regulation.

By contrast, looking at our results of $\mathrm{ONH}$ blood flow there is no indication of any significant difference between dopamine and placebo. According to Hayreh, ${ }^{23}$ the main vascular supply for the superficial layers of the $\mathrm{ONH}$ descents from retinal arterioles. Hence, dopamine modulations of LDF parameters in the ONH might be expected from our retinal results, because animal experiments indicate that the superficial layers of the ONH do significantly contribute to the signal. ${ }^{24}$ However, high interindividual differences of vascular supply to this region exist, and even different sectors of the ONH can have a different supply. ${ }^{25}$ In addition, the exact depth of the measurement with LDF in the ONH is unknown. In a monkey experiment, cutting the ciliary posterior arteries did not modify the LDF parameters. ${ }^{24}$ This has been cited as evidence that the deeper layers of the $\mathrm{ONH}$ vasculature do not contribute to the LDF signal. ${ }^{26}$ By contrast, ligation of the central retinal artery reduced $\mathrm{ONH}$ blood flow by only $40 \%$ in the same set of experiments, indicating that $60 \%$ of the flow remained unaffected by stopping flow to the superficial layers. ${ }^{24}$ This is in keeping with in vitro data indicating that LDF can penetrate deep enough to measure blood flow down to the level of the lamina cribrosa. ${ }^{27}$ Interestingly, the data obtained in the present study indicating increased blood flow in the retina, but not in the ONH after dopamine infusion show some similarity to previous data in cat, where an increase in retinal, but not ONH perfusion was observed after administration of the calcium channel blocker nicardipine. ${ }^{28}$ This again indicates that LDF performed in the $\mathrm{ONH}$ does not simply reflect perfusion in the superficial $\mathrm{ONH}$ capillaries. $^{29}$

\section{The role of dopamine on ocular blood flow regulation}

Dopamine is known to have a crucial role in the light adaptational pathway rewiring of the visual afferents. ${ }^{30-32}$ In addition there is evidence for a close morphological association of dopaminergic cell processes with blood capillary walls in monkeys and rats. ${ }^{33}$ Our study does not provide any evidence as to which dopamine receptor subtypes are involved in the modulation of retinal vascular tone or if other catecholamine receptors have an impact as well. In conclusion, however, our data suggest a distinct dopaminergic contribution to retinal perfusion. To elucidate the regulatory role and significance of this modulator in vivo requires more detailed further examination.

\section{ACKNOWLEDGEMENTS}

The authors would like to thank Karin Urstöger for support with subject management and Anna Bielik for technical assistance with the HPLC measurements.

\footnotetext{
Authors' affiliations

Karl-Heinz Huemer, Claudia Zawinka, Gerhard Garhöfer, Elisabeth

Golestani, Departments of Clinical Pharmacology, Medical University of Vienna, Vienna A-1090, Austria

Brigitte Litschaver, Departments of Physiology and Pathophysiology,

Medical University of Vienna, Vienna A-1090, Austria

Guido T Dorner, Departments of Ophthalmology, Medical University of Vienna, Vienna A-1090, Austria

Leopold Schmetterer, Departments of Biomedical Engineering and Physics, Medical University of Vienna, Vienna A-1090, Austria

Competing interests: None declared.

\section{REFERENCES}

1 Chiou GCY, Chen YJ. Effects of dopamine agonist, bromocriptine, and some dopamine antagonists on ocular blood flow. J Ocul Pharmacol 1992;8:285-94.

2 Hong S-J, Chiou GCY. Effects of dopamine agonists and antagonists on pulsatile blood flow of ocular hypertensive rabbits. J Ocul Pharmacol 1993;9:1 17-24.

3 Potter DE, Crosson CE, Heath AR, et al. Alpha 2 and DA2 agonists as antiglaucoma agents: comparative pharmacology and clinical potential. J Ocul Pharmacol 1990;6:251-7.
} 
4 Prünte C, Nuttli I, Markstein R, et al. Effects of dopamine D-1 and D-2 receptors on intraocular pressure in conscious rabbits. J Neural Transm 1997; 104:111-23.

5 Huemer K-H, Garhöfer G, Zawinka C, et al. Effects of dopamine on human retinal vessel diameter and its modulation during flicker stimulation. Am J Physiol Heart Circ Physiol 2003;284:H358-63.

6 Smedes F, Kraak JC, Poppe H. Simple and fast solvent extraction system for selective and quantitative isolation of adrenaline, noradrenaline and dopamine from plasma and urine. J Chromatogr 1982;231:25-39.

7 Opacka-Juffry J, Tacconelli F, Coen CW. Sensitive method for determination of picogram amounts of epinephrine and other catecholamines in microdissected samples of rat brain using liquid chromatography with electrochemical detection. J Chromatogr 1988;433:41-51.

8 Schmetterer L, Lexer F, Unfried C, et al. Topical measurement of fundus pulsations. Optic Eng 1995;34:711-16.

9 Schmetterer L, Dallinger S, Findl, O, etal. A comparison between laser interferometric measurement of fundus pulsation and pneumotonometric measurement of pulsatile ocular blood flow. 1. Baseline considerations. Eye 2000;14:39-45.

10 Lieb WE, Flaharty PM, Sergott RC, et al. Color Doppler imaging provides accurate assessment of orbital blood flow in occlusive carotid artery disease. Ophthalmology 1991;98:548-52.

11 Riva CE, Grünwald JE, Sinclair SH, et al. Blood velocity and volumetric flow rate in human retinal vessels. Invest Opthalmol Vis Sci 1985;26:1124-32.

12 Riva CE, Harino S, Petrig BL, et al. Laser Doppler flowmetry in the optic nerve. Exp Eye Res 1992;55:499-506.

13 Chiou GCY, Chen YJ. Effects of antiglaucoma drugs on ocular blood flow in ocular hypertensive rabbits. J Ocul Pharmacol 1993;9:13-24.

14 Reitsamer HA, Zawinka C, Branka M. Dopaminergic vasodilation in the choroidal circulation by d1/d5 receptor activation. Invest Ophthalmo Vis Sci 2004;45:900-905.

15 Prielipp RC, Wall MH, Groban L, et al. Reduced regional and global cerebral blood flow during fenoldopam-induced hypotension in volunteers. Anesth Analg 2001;93:45-52.

16 Amenta F, Ferrante F, Ricci A. Pharmacological characterisation and autoradiographic localisation of dopamine receptor subtypes in the cardiovascular system and in the kidney. Hypertens Res 1995; 18(Suppl 1):23-7.

17 Missale C, Nash SR, Robinson SW, et al. Dopamine receptors: from structure to function. Physiol Rev 1998;78:189-225.
18 Veruki ML, Wässle H. Immunohistochemical localization of dopamine D1 receptors in rat retina. Eur J Neurosci 1996;8:2286-97.

19 Wu DM, Kawamura H, Li Q, et al. Dopamine activates ATP-sensitive $\mathrm{K}^{+}$currents in rat retinal pericytes. Vis Neurosci $2001 ; 18: 935-40$.

20 Jandrasits K, Luksch A, Söregi A, et al. Effect of noradrenaline on retinal blood flow in healthy subjects. Ophthalmology 2002;109:291-5.

21 Reitsamer HA, Kiel JW. Effects of dopamine on ciliary blood flow, aqueous production, and intraocular pressure in rabbits. Invest Ophthalmol Vis Sci 2002;43:2697-703.

22 Schmetterer L, Dallinger S, Findl O, et al. A comparison between laser interferometric measurement of fundus pulsation and pneumotonometric measurement of pulsatile ocular blood flow. 2. Effects of changes in $\mathrm{pCO}_{2}$ and $\mathrm{pO}_{2}$ and of isoproterenol. Eye 2000;14:46-52.

23 Hayreh SS. Blood supply of the optic nerve head. Ophthalmologica 1996;210:285-95.

24 Petrig BL, Riva CE, Hayreh SS. Laser Doppler flowmetry and optic nerve head blood flow. Am J Ophthalmo 1999;127:413-25.

25 Hayreh SS. Blood flow in the optic nerve head and factors that may influence it. Prog Ret Eye Res 2001;20:595-624.

26 Hayreh SS. The blood supply of the optic nerve head and the evaluation of it myth and reality. Prog Ret Eye Res 1996;20:563-93.

27 Koelle JS, Riva CE, Petrig BL, et al. Depth of tissue sampling in the optic nerve head using laser Doppler flowmetry. Lasers Med Sci 1993;8:49-54.

28 Harino S, Riva CE, Petrig BL. Intravenous nicardipine in cats increases optic nerve head but not retinal blood flow. Invest Ophthalmol Vis Sci 1992;33:2885-90.

29 Piltz-Seymour JR. Laser Doppler flowmetry of the optic nerve head in glaucoma. Surv Ophthalmol 1999;43(Suppl 1):191-8.

30 Baldridge WH, Ball AK, Miller RG. Dopaminergic regulation of horizontal cell gap junction particle density in goldfish retina. J Comp Neurol 1987;265:428-36.

31 MacGuire G, Hamasaki DI. The retinal dopamine network alters the adaptational properties of retinal ganglion cells in the cat. J Neurophys 1994;72:730-41.

32 Xin D, Bloomfield SA. Dark and light-induced changes in coupling between horizontal cells in mammalian retina. J Comp Neurol 1999;405:75-87.

33 Favard C, Simon A, Vigny A, et al. Ultrastructural evidence for a close relationship between dopamine cell processes and blood capillary walls in Macaca monkey and rat retina. Brain Res 1990;523:127-33.

\section{Access a vast information database with Toll-Free linking}

"Toll-free" linking gives you immediate access to the full text of many of the cited articles in a paper's reference list-FOR FREE. With the support of HighWire's vast journal catalogue, a huge reference library is now open to you. If HighWire hosts the journal, you can view the full text of the referenced article, completely free of charge by following the Free Full Text links in the references. 